\title{
The Present Situation and Countermeasures of Training Simulation
}

\section{System}

\author{
Qinxin Meng ${ }^{1, a}$, Qinlei Meng ${ }^{2, b}$ \\ ${ }^{1}$ China Academy of Electronics and Information Technology, Beijing City, 100041, China \\ ${ }^{2} 760$ Institute, Second Academy of China Aerospace Science \& Industry Corp, Beijing City, \\ 100854, China
}

Key words: Training simulation; Development status; Development strategy

\begin{abstract}
Simulation training is a training method in recent years with the development of equipment. The technology is complex, expensive, and high requirement on the operation, so the modern weapons should not make real training compared with rifles and other small arms. Therefore, to strengthen the research and development of simulation training system and increase training simulation in military training proportion are one of the most primary tasks in military training transformation. Based on the author's study and practical experience, this paper firstly analyzed the current situation on developing training simulation system at home and abroad, and then discussed the trend for the training simulation system and the countermeasures for developing the simulation training system in China.
\end{abstract}

\section{Introduction}

In order to master the operation skills by the operators, coordinate the training in peacetime and operations in wartime, reduce training costs, improve the quality of training, an urgent problem with the help of simulation technology to provide simulation equipment consistent with wartime operation must be solved. Under such situation, the research in the field of training simulation is now coming into being. Simulation training equipment being applied in equitment operation simulation is very commonly seeing now. Generally speaking, self-propelled artillery driving simulator, fire simulator and tactical training simulation system are the concrete manifestations on training simulation researches. Therefore, specifically, simulation training is a kind of imitation by using the similarity principle and the modern simulation technology, which simulates the various properties of the objective entity including the appearance, so as to provide training method for improving the skills of the users.

\section{The State of Development for Training Simulation System at Home and Abroad}

Many military developed countries headed by the United States, have developed many mature training simulation systems, of which the typical one is the cooperative tactical trainer for the U.S. Army; it will meet the training needs to the simulation system connections in different functional areas so as to form a multi-arm service combating for the virtual battlefield environment; the air combat simulation system developed by the United States Air Force is mainly used in the training tool on skills training, free combat and command when in execution of joint and cooperative operations; the U.S. Air Force Laboratory AFRL has developed the project tool for air defense drill; the U.S. Army' s joint "four items" electronic warfare training simulation system is mainly used in tactical air combat and air defense simulating in electronic warfare environment. Navy combat simulation system reflects the the tactics as a trend in the strategy after decision-making and action mainly through the process of simulating the real 
combat to trainees so as to test the training goal. The "peeping-90" manoeuvre organized by the U.S. Army before the Gulf War simulates naval operations by using the ENWGS system. In addition, the toolbox and generation environment of the VPI company in Canada, the virtual force in MAK company, which overally regard the the establishment and operation of scenario as main lines.

The main features of training simulation system in Europe and the United States are manifested in the following aspects: the first is strengthening the system software modular, which has a regular datum format, high portability and reusability; the system interface technology is unified, the software model is easy to be extended with good coupling performance and arm training simulation system has a strong compatibility and interoperability. For example, the U.S. Army has made modeling and simulation plan with the definition of the plan in 1995; a clear goal for the modeling and Simulation also has been put forward, and the first item of the goal is to provide public general technical framework of modeling and simulation, including the three sub-goals: high level architecture framework, functional description of mission space and the data standard, which greatly improve the versatility, reusability, sharing and interoperability of the simulation system. Second, all kinds of databases are relatively complete, especially for the terrain database, tactical and technical database of all kinds of weapons and equipments, and the model is based on evaluating the operational effectiveness of weapon equipment; the third is a high degree of simulation. All kinds of evaluation models' computing methods developed by U.S. Army on combat effectiveness are perfected and tested in the Gulf War and the Iraq war, etc; fourth, the user interface on system is friendly and easy to be operated. The training simulation system has become an important tool for military training in Europe and the United States. It is relatively late to the development and application of Chinses army's training simulation system, most of which have learned principles of the foreign military simulation training systems. There is still a gap in such aspect compared with developed countries. The gap is mainly showed on the developed training simulation system, which is still in the small scale simulation training, so as to still exists the certain gap between the efficiency evaluation and the actual situation.

\section{The Development Trend of Training Simulation System}

The modeling should be complex, holographic, accurate, interactive. Under the circumstance of informatization, the war shows three characteristics: non- symmetric, non-linear and non-contacting, which also determine the research on modern simulation training being based on the three characteristics. It fundamentally promotes and guides the expansion of the modeling operation theory from the "linear theory" to "non-linear theory". Accordingly, the modeling theory is deepened from simplicity to complexity, which means transforming the simple and linear system modeling into a complex and non-linear one. Secondly, the future modeling focus is to build a high fidelity synthetic battlefield, and we must expand simulation to the weapon's external characteristics on functions and performances into the simulation to the internal characterisctis on systems and mechanisms of troops and organizations, which will highlight the realistic description on the complexity and dynamic of the real world as well; we'd like to conduct a comprehensive simulation on military organization, tactics and battlefield, so as to show the holographic characteristics.

The application of high-tech means will improve the authenticity of the simulation system. The development of the training simulation depends on the improvement of supporting technology to a large extent. Therefore, the rapid development for the technologies on the fields of computer, simulation, multimedia, artificial intelligence, system engineering, sensor and the three-dimensional imaging will let the training simulation system come to a new level. For example, the development of computer network technology realizes all kinds of remote training 
simulation systems.

Simulation changes its direction into humanization and enjoyment. The training simulation system of enjoyment and humanization provides a real and operative environment. A friendly interface with high visibility can arouse the trainees' emotions and it also improve the initiative and training effects. The development of virtual simulation technology simulates realistic training environment so as to provide a good training environment for trainees. Further, the componentization represents the trend of the modularization, which shortens the software development cycle and improves development efficiency.

\section{Countermeasures to the Development of Training Simulation System in China}

Further strengthen the simulation of complex information environment. With the extensive application of information technology on the battlefield, especially at this stage, there is an essential difference between the future battlefield and the traditional battlefield under the conditions of informatization; the battlefield has been greatly expanded from the land, sea, air, space in a traditional sense to the electromagnetic space, cyberspace and human cognitive space; the information environments formed by the electromagnetic space, a network space and human cognitive space have become the main parts of the battlefield, which will greatly increase the complexity of the battlefield. As the battlefield space,the electromagnetic space, network space and cognitive space have not been understood comprehensively by the public for the spaces are the relative latecomers and informationzlized products. However, information battlefield plays an important role in the future battlefield, which will lead to revolutionary changes on operational pattern, command, control and collaborative troops. To understand and stimulate these new battle spaces is a precondition for the future combat simulation environment, and it is also an important guarantee for grasping the essence, law and developing direction. To grasp the essence of the war in the future, we must regard the important guarantees from the law and the development direction as the important means to improve students future combat capabilities under complex environmental conditions. Therefore, the future research should be focused on how to describe these new combat spaces, strengthening the simulation on complex information environment and assessing impacts on the operations and combat effectiveness.

Improve the standardization of the data format in the training simulation system and the reusability of the model. We focus the future training simulation system on the formation and promotion of combined operational capabilities with the system confrontation simulation. To achieve the combined and cooperative combat by multi-services and arms, we need a large number of data and models. Therefore, the future development for training simulation system will regard the open and public database as the foundation, and strengthen the unified data format, model reusability and compatibility, which can greatly improve the utilization rates of the data and the models, shorten the construction period of the simulation training system, and can increase the credibility of the simulation training. To realize it, we should implement from the following aspects: first, we must standardize the data types and technical frameworks; second, all kinds of models and databases should be extensive and open; third, we should do well in the collection of all kinds of data.

Support command agencies at all levels to parallelly develop operational plans. At the most of existing training simulation systems, plan is consistent with the traditional mode of operation. Under the condition of informatization, joint operations is sudden, quick and complex, which puts forward higher requirement on the timeliness of operational plans. The timeliness at all levels of commands the traditional operation mode in serial plans is poor. In the informatizaon battlefield, such as the use of parallel plan, can greatly shorten the operational planning cycle, reduce the time on decision cycle, speed up the rhythm from information to 
movement; further, quickly making and implementing an operation plan through dynamic and parallel way can shorten the reaction time to fully grasp the combat opportunity and improve operational efficiency. The battlefield information system not only provides the general situation maps based on the battlefield resources of public information, but also provides the force and resource databases in different resolutions so that bring powerful spport for command agencies at all levels when making plans parallelly. This change will lead to a requirement on the future training simulation system; namely, it must be equipped with the performance to support command agencies at all levels when making combat plan parallelly.

\section{Reference}

[1] Zong Zhaowen, Qin Hao, Xu Lei, Mao Changxue, Du Guofu, Shen Yue, Guo Qingshan. Progress and Enlightenment on Simulated Training of Rescue Technology Training [J]. Southern China Journal of National Defense Medicine, 2016, (08): 526-528.

[2] Jiao Kaizhe, Cheng Peiyuan, Liu Tao, Meng Chao. Research on Foreign Military Virtual Training System [J]. Flight and Missile, 2013, (06): 60-63+67.

[3] Qu Yang, Li Peilin, Wang Wei, Xu Xiaodong, Yang Chenglong. The status and trend of U.S. Virtual Training [J]. Flight and Missile, 2013, (05): 24-28.

[4] An Xing, Li Gang, Xu Linwei, Shi Ying. Current Application and Development of Virtual Practical Technology in the U.S. Army Simulation Training [J]. Electro- optics and Control, 2011, (10): 42-46.

[5] Liu Xingyan, Liu Shibin. Application and Trend of Military Training Simulation [J]. Network and Information, 2010, (04): 32

[6] Zhang Jinrong. Some Thoughts on How to Promote the Leaping Development of Naval Simulation Training [J]. Military Operations and Systematic Engineering, 2010, (01): 44-47.

[7] Ding Weirong. The Current Status of Military Simulation Equipment at Home and Abroad [J]. Applied Optics, 1987, (06): 61-66+72. 